\title{
O que sabemos sobre a violência contra as mulheres em tempos pandêmicos? Insights baseados em tendências de busca
}

What do we know about violence against women in pandemic times? Insights based on search trends

¿Qué sabemos sobre la violencia contra las mujeres en tiempos de pandemia? Estadísticas basadas en tendencias de búsqueda

Rafaela Ferreira Guatimosim https://orcid.org/0000-0002-07661827

Ana Luiza Silva Teles - https://orcid.org/0000-0002-0438-3577

Fabiano Franca Loureiro - https://orcid.org/0000-0002-4691-4495

Leonardo Rodrigo Baldaçara - https://orcid.org/0000-0002-5201-8515

Antônio Geraldo da Silva - https://orcid.org/0000-0003-3423-7076

Débora Marques de Miranda - https://orcid.org/0000-0002-7081-8401

Leandro Fernandes Malloy-Diniz - https://orcid.org/0000-0002-6606$\underline{1354}$

\section{RESUMO:}

Objetivo: Mulheres tem sido associadas, desde o início da pandemia com níveis mais elevados de stress e ansiedade. Esta breve comunicação visa avaliar a situação da violência doméstica contra a mulher no Brasil durante o isolamento social devido à pandemia da COVID-19. Métodos: Extraímos dados do Google Trends mostrando a magnitude das pesquisas sobre os temas violência doméstica e denúncias de violência doméstica e depois comparamos com dados de relatórios de denúncias. Resultados: As buscas no Google contendo esses termos aumentaram enquanto as denúncias contra a violência doméstica diminuíram. Conclusão: 0 crescimento das buscas sobre violência doméstica e queixas de violência doméstica indica a possibilidade de um aumento real deste tipo de violência no Brasil. 
Palavras chave: Violência doméstica, Tendências de busca, COVID-19

\section{ABSTRACT:}

Objective: Since the beginning of the pandemic, women have been associated with higher levels of stress and anxiety. This short communication aims to assess the situation of domestic violence against women in Brazil during social isolation caused by the COVID-19 pandemic. Methods: We extracted data from Google Trends showing the magnitude of searches on the topics domestic violence and reporting domestic violence and then compared with the data on actual reports of domestic violence. Results: Searches on Google containing those terms have increased while the number of reports of domestic violence have decreased. Conclusion: The increase in internet searches about domestic violence and how to report domestic violence indicates the possibility of a real rise in this type of violence in Brazil.

Keywords: Domestic violence, search trends, COVID-19.

\section{RESUMEN:}

Objetivo: Desde el comienzo de la pandemia, las mujeres se han asociado con niveles más altos de estrés y ansiedad. Esta breve comunicación tiene como objetivo evaluar la situación de la violencia doméstica contra las mujeres en brasil durante el aislamiento social causado por la pandemia de covid-19. Métodos: extrajimos datos de Google Trends que muestran la magnitud de las búsquedas sobre los temas de violencia doméstica y denuncia de violencia doméstica y luego los comparamos con los datos sobre informes reales de violencia doméstica. Resultados: Las búsquedas en Google que contienen esos términos han aumentado, mientras que el número de denuncias de violencia doméstica ha disminuido. Conclusión: El aumento de las búsquedas en internet sobre la violencia doméstica y cómo denunciar la violencia doméstica indica la posibilidad de un aumento real de este tipo de violencia en brasil.

Palabras clave: Violencia doméstica, Tendencias de búsqueda, COVID19. 
Como citar: Guatimosim, R.F; Teles, A.L.S; Loureiro, F.F.; Baldaçara, L.R.; Silva, A.G.; Miranda, D.M.; Malloy-Diniz, L.F. - O que sabemos sobre a violência contra as mulheres em tempos pandêmicos? Insights baseados em tendências de busca. Debates em Psiquiatria, Rio de Janeiro, 2021; 11:1-14. https://doi.org/10.25118/2763-

9037.2021.v11.204

Conflito de interesses: declaram não haver

Fonte de financiamento: declaram não haver

Parecer CEP: não se aplica

Recebido em: 06/08/2021

Aprovado em: 08/08/2021

Publicado em: 26/09/2021

\section{Introdução}

O sofrimento psicológico tem sido considerado entre os resultados mais importantes da pandemia de COVID-19 [1]. Como apontado por Connor et al. [2], disparidades de gênero nos riscos e consequências para a saúde provavelmente serão ampliadas durante a pandemia de COVID-19. As mulheres, desde o início da pandemia, têm sido associadas a um maior impacto psicológico e a níveis mais elevados de estresse, ansiedade e depressão, como demonstrado no estudo realizado por Wang et al. []ㅡ.

Há vários fatores que podem aumentar o impacto das pandemias na saúde mental da mulher, o que inclui desigualdades de gênero que fortalecem a sobrecarga de trabalho em casa e a violência de gênero [4].

Em uma recente revisão sistemática, Piquero et al. [5] analisou 37 estudos e descobriu que pelo menos 29 relataram uma associação estatisticamente significativa entre a permanência na política de "fique em casa" e o aumento da violência doméstica. Nesse contexto, a Organização das Nações Unidas (ONU) alertou sobre esse problema, destacando a necessidade de promover estratégias de proteção para as mulheres $[\underline{6}, \underline{7}]$.

Essa situação é alarmante, uma vez que as vítimas de violência doméstica são frequentemente encontradas em situações pessoais imprevisíveis, que tornam quase impossível a tomada de medidas legais eficazes []․ No 
estudo realizado por Artz []ㅡ, no qual ela entrevistou 503 mulheres, foi demonstrado que o medo do perpetrador e a coerção para retirar ações legais foram fatores significativos que afetaram as decisões das mulheres de retornar ao tribunal.

Artz [] também informa que dos milhares de mulheres que iniciam o processo de obtenção de ordens de proteção nos EUA, menos da metade retorna ao tribunal para obter os pedidos finais.

Para melhor examinar o cenário brasileiro em relação à situação da mulher, foi utilizado o Google Trends, uma ferramenta de acesso livre, que já foi utilizada por diversas pesquisas científicas [ㅁ-11].

No estudo conduzido por Vosen \& Schmidt [11], as tendências de busca do Google provaram ser melhores do que os indicadores convencionais baseados em questionários em quase todos os experimentos realizados em pesquisas relacionadas ao consumo.

Na revisão sistemática de Tsao et al. [10], foi mostrado que as pesquisas do Google Trends de 2 semanas antes podem ser usadas para modelar o número de casos de COVID-19. Há evidências de que a ferramenta é útil para abordar fenômenos relacionados à saúde e é até mesmo reconhecida como promissora pelo Instituto de Medicina, de forma a complementar as pesquisas e descobertas atuais [ㅇ].

Além disso, os dados possibilitados pelo Google Trends fornecem informações relevantes sobre o comportamento da população, já que as tendências da pesquisa podem expor e ajudar a prever os fenômenos sociais $[\underline{9}, 11]$. Esse aspecto preditivo também é útil, uma vez que os comportamentos verificados podem reaparecer no futuro sob condições ou contingências similares.

Tal cenário destaca a urgência desta comunicação breve sobre violência doméstica contra a mulher e serve como um incentivo para que políticas públicas sejam criadas e/ou reforçadas, a fim de elaborar mecanismos de prevenção e cuidado para as vítimas. Nosso estudo visa investigar mais a fundo as tendências da pesquisa, a fim de melhor examinar e abordar a violência doméstica em um contexto pandêmico. 


\section{Método}

Em 28 de fevereiro de 2021, foram divulgados dados do Google Trends em comemoração ao Dia Internacional da Mulher. Optamos por pesquisar termos em português relacionados a temas de violência contra a mulher.

Fizemos o download dos dados para as seguintes entradas de pesquisa: [como denunciar + violência doméstica] e [violência doméstica] no dia 05/04/2021. Os dados coletados no Brasil foram de 2004 a 2021, sem especificar a categoria da consulta.

O Google Trends é uma ferramenta que fornece dados de interesse de busca em relação a seu ponto mais alto. O valor de 100 representa o pico de popularidade do termo. Isso significa que os dados são indexados de modo que 100 representa o maior interesse de pesquisa, e as pontuações adicionais são feitas proporcionalmente em relação ao ponto máximo.

Descrevemos as frequências de interesse de pesquisa anualmente ao longo do tempo, e depois comparamos os dados de tendências de pesquisa com os relatórios sobre crimes contra a mulher do Fórum Brasileiro de Segurança Pública (FBSP) [12].

\section{Resultados}

No Brasil, em 2020, o número de buscas por como denunciar + violência doméstica atingiu o seu ponto mais alto nos últimos 17 anos, assim como o interesse pela violência doméstica, que também atingiu seu ponto mais alto nos últimos 11 anos [티]

O auge do interesse de busca de como denunciar violência doméstica foi atingido em março de 2020, o primeiro mês de extensas medidas de distanciamento social na maioria dos estados do Brasil. Também é digno de nota o resultado de maio do mesmo ano, quando um total de 98 foi alcançado. O ponto mais alto de interesse de busca por violência doméstica foi alcançado em julho de 2020, quando um total de 28 foi registrado.

A Figura 1 exibe os dados do Google Trends sobre o interesse de busca pelos termos violência doméstica e como denunciar + violência doméstica no Brasil de 01/03/2015 a 04/05/2021. A linha pontilhada marca o mês de fevereiro, o mês em que o início da Emergência de Saúde Pública de Importância Nacional (ESPIN) foi demarcado no país como resultado da COVID-19, declarada através da Portaria no 188/GM/MS. 


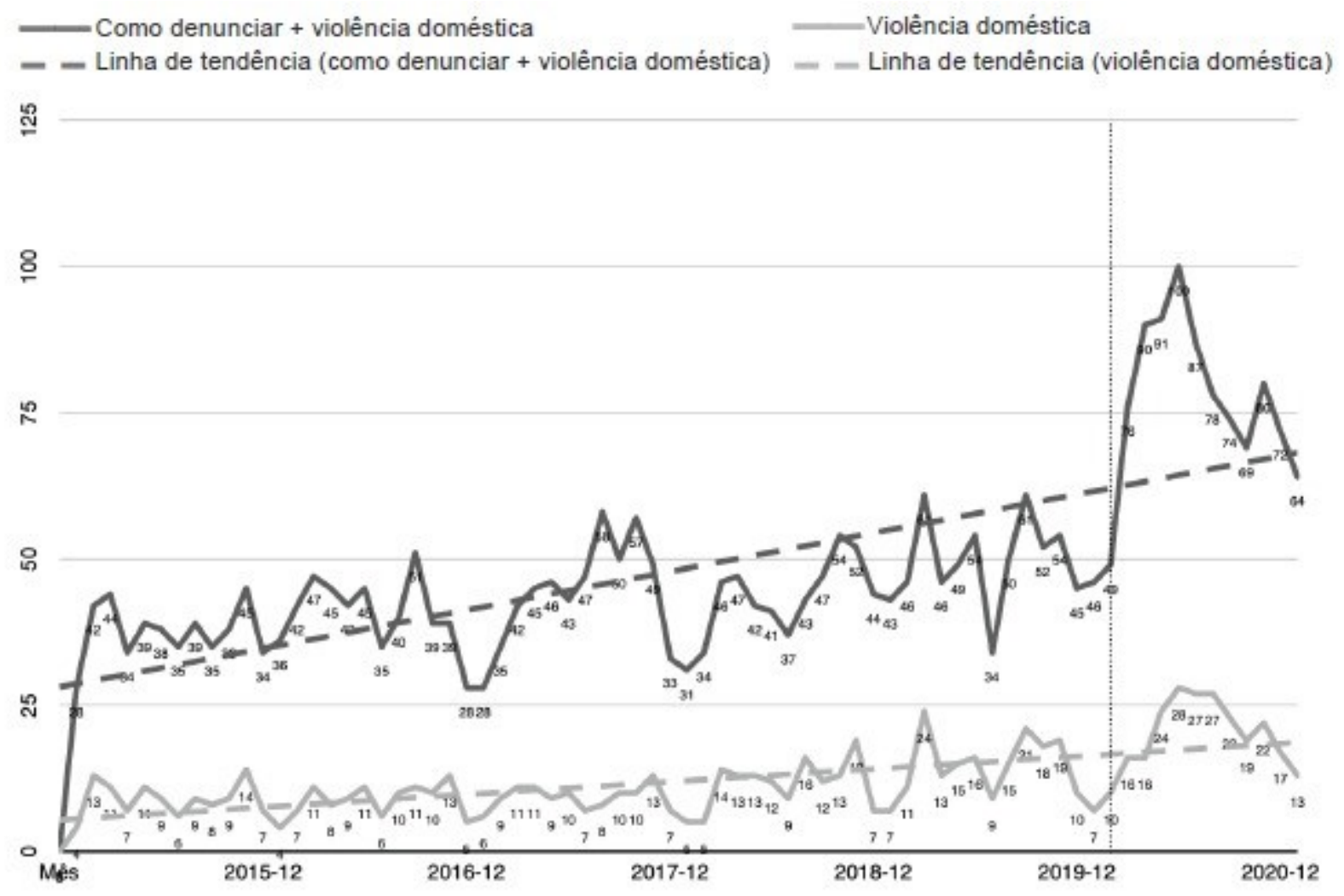

Figura 1 - O gráfico mostra duas curvas: a de denúncia + violência doméstica e a de violência doméstica. Também exibe a linha de tendência de cada uma dessas curvas. É apresentada uma linha pontilhada que marca o momento em que foi declarado no Brasil o estado de pandemia.

\section{Discussão}

Esses dados mostram um interesse maior em como denunciar do que na busca de o que é violência doméstica. Revelam também um aumento nas buscas sobre violência doméstica e contrastam com os dados coletados pelo Fórum Brasileiro de Segurança Pública (FBSP), que declaram uma redução em uma série de crimes contra a mulher [12].

Segundo a FBSP, o crime de lesão corporal teve uma redução em quase todos os estados brasileiros, mostrando uma queda de $9,9 \%$ quando se compara o primeiro semestre de 2020 com o de 2019. Houve também uma redução dos crimes de ameaça em 15,8\%.

Os registros de estupro caíram $23,8 \%$ no mesmo período. Entretanto, em contraste com os outros dados apresentados, o feminicídio teve um aumento de $1,9 \%$ no mesmo período. 
Outro dado que chama a atenção é o aumento de $3,8 \%$ no número total de chamadas para 190 registradas sob a natureza da violência doméstica no primeiro semestre, quando comparado com o de 2019.

A isso devemos acrescentar que, como declarado por Artz [ㅈ], a denúncia de violência doméstica pode ter um efeito boomerang, em que procurar ajuda amplifica comportamentos ameaçadores por parte do agressor. Artz [8] explica que, ao contrário da maioria dos tipos de crime, o perpetrador é motivado a retaliar quando a vítima procura ajuda jurídica. No entanto, as vítimas precisam ser conscientizadas de que a intensidade e a brutalidade da violência doméstica aumentam com o tempo e o abuso psicológico e mental se transforma em violência física []].

Dado este quadro complexo, no qual a busca por denúncias está aumentando e os registros de violência estão diminuindo, torna-se evidente a necessidade de atenção à situação em que as mulheres brasileiras se encontram.

Embora as medidas de isolamento social sejam extremamente importantes e necessárias para a gestão da pandemia, as recomendações de ficar em casa reforçaram a convivência com os agressores potenciais, como foi relatado por vários estudos [ㅁ].

Além de limitar o seu acesso às redes de proteção, as restrições de viagem têm impedido e/ou dificultado a saída das vítimas do ambiente violento, bem como, em muitos casos, impedindo-as de ter acesso a familiares, abrigos, casas de abrigo temporário e hotéis. Ademais, diante do atual período estressante, há uma tendência de agravamento das tensões familiares, especialmente naqueles com histórico de violência doméstica recorrente, que se tornam ainda mais propensos a tais situações [14].

Destacamos também um fato importante: em 2020, a taxa de desemprego no país aumentou substancialmente, e a taxa de desemprego entre as mulheres foi de $16,4 \%$ no último trimestre do ano, de acordo com os dados do IBGE [15], o que acaba refletindo em outros problemas, como a dependência econômica e, com isso, uma maior vulnerabilidade ao agressor. Além disso, durante a pandemia, muitas mulheres acabaram ficando sobrecarregadas devido ao acúmulo de tarefas, tanto entre as mulheres com emprego remunerado quanto entre as que não o têm, que 
também acabam acumulando mais carga de trabalho doméstico e cuidando de outras pessoas, como crianças e familiares [16].

De acordo com Dang \& Viet Nguyen [17], a pandemia de COVID-19 aumentou a diferença de gênero no mercado de trabalho, uma vez que muitas mulheres ficaram desempregadas e tiveram uma redução de renda esperada em comparação com os homens. As mulheres em vulnerabilidade financeira são mais propensas a serem vítimas de violência do parceiro (Pereira \& Gaspar) [18], e os autores podem supor que, nesses cenários de dependência financeira, a denúncia de violência doméstica às autoridades se torna menos provável.

Há um cenário em que o feminicídio (um crime que é mais difícil de esconder) aumentou, assim como as chamadas à polícia e as pesquisas sobre como denunciá-lo, enquanto os registros de vários crimes associados à violência doméstica diminuíram [11]. Ainda, há também o fato de um aumento global dos crimes de violência doméstica associados à pandemia ter sido relatado pela ONU $[\underline{6}, \underline{7}]$.

A partir desse cenário, especula-se que o isolamento social contribui para a subnotificação das estatísticas e que as mulheres estão enfrentando maiores dificuldades para fazer denúncias e acessar serviços especializados para mulheres. Essa hipótese destaca a necessidade de mais pesquisas sobre o contexto atual, no qual as mulheres brasileiras se encontram, e de mais apoio para tornar possível a apresentação de denúncias.

Este estudo apresenta algumas limitações importantes. Os dados de diferentes fontes foram cruzados, o que já pode levar a enviesamentos ou imprecisões na análise. Também reconhecemos que essas estatísticas envolvem variáveis diferentes, e atribuir um comportamento ou efeito a apenas uma dessas variáveis também gera imprecisões.

Embora reconhecendo as possíveis falhas, ainda se aponta que a hipótese levantada a partir dos dados é alarmante. O objetivo desta breve comunicação é chamar a atenção para essa hipótese, que pelo simples fato de ser possível requer mais estudos.

Um dos pontos fortes do estudo consiste no fato de que existem dados indiretos que podem refletir uma abordagem menos suscetível à notificação. 
Portanto, os serviços de proteção às mulheres devem ser redirecionados para ações que possam ser eficazes mesmo remotamente, tais como campanhas que evidenciem a existência, importância e eficácia dos canais de denúncia: os números 180 e 100, estimulando a mulher a denunciar, assim como pessoas próximas a ela que vejam o que está acontecendo.

Também é possível denunciar a violência através do aplicativo Proteja Brasil, desenvolvido pelo Fundo das Nações Unidas para a Infância (UNICEF) e pela então Secretaria de Direitos Humanos da Presidência da República (hoje Ministério dos Direitos Humanos), o que permite não só fazer a denúncia diretamente através do aplicativo, mas também informar a localização dos órgãos de proteção nas principais capitais, além de conter também informações sobre as diferentes violações.

Além disso, com base nas informações e números encontrados no Google Trends, também é importante divulgar os comportamentos que caracterizam a violência doméstica para um maior conhecimento da população, já que, com exceção da violência física e sexual, os demais podem se manifestar de forma muito sutil, já que não deixam marcas visíveis.

\section{Conclusão}

Diante de um quadro em que o feminicídio aumentou, assim como as chamadas à polícia e as pesquisas sobre como denunciá-lo, enquanto os registros de vários crimes associados à violência doméstica diminuíram, destaca-se a necessidade de chamar a atenção às mulheres.

A partir desse cenário, especula-se que o isolamento social contribui para a subnotificação das estatísticas de denúncia e que as mulheres estão enfrentando maiores dificuldades para fazer reclamações e acessar serviços especializados para elas. Tal hipótese reforça a necessidade de mais pesquisas sobre o contexto atual em que as mulheres brasileiras se encontram e de prestar assistência para tornar possível a apresentação de denúncias. 


\section{Referências}

1. Silva AG, Miranda DM, Diaz AP, Teles AL, Malloy-Diniz LF, Palha AP. Mental health: why it still matters in the midst of a pandemic. Braz J Psychiatry. 2020;42:229-31. https://doi.org/10.1590/1516-4446-20200009 PMid:32267344 PMCid:PMC7236155

2. Connor J, Madhavan S, Mokashi M, Amanuel H, Johnson NR, Pace LE, et al. Health risks and outcomes that disproportionately affect women during the Covid-19 pandemic: a review. Soc Sci Med. $2020 ; 266: 113364$.

https://doi.org/10.1016/j.socscimed.2020.113364 PMid:32950924 PMCid:PMC7487147

3. Wang C, Pan R, Wan X, Tan Y, Xu L, Ho CS, et al. Immediate psychological responses and associated factors during the initial stage of the 2019 Coronavirus disease (COVID-19) epidemic among the general population in China. Int J Environ Res Public Health. 2020;17:1729. https://doi.org/10.3390/ijerph17051729 PMid:32155789 PMCid:PMC7084952

4. Souza AS, Souza GF, Praciano GA. Women's mental health in times of COVID-19. Rev Bras Saude Mater Infant. 2020;20:659-61. https://doi.org/10.1590/1806-93042020000300001

5. Piquero AR, Jennings WG, Jemison $E$, Kaukinen $C$, Knaul FM. Domestic violence during the COVID-19 pandemic - evidence from a systematic review and meta-analysis. J Crim Justice. 2021;74. Epub 2021 Mar 9. https://doi.org/10.1016/j.jcrimjus.2021.101806

6. Guterres A This is the moment to step up for the vulnerable [Internet]. 2020 Mar 23 [cited 2021 Apr 16].

http://www.un.org/en/un-coronavirus-communicationsteam/moment-step-vulnerable

7. United Nations Population Fund (UNFPA). COVID-19: a gender lens: protecting sexual and reproductive health and rights and promoting gender equality. New York: UNFPA; 2020. https://www.unfpa.org/sites/default/files/resource-pdf/COVID19 A Gender Lens Guidance Note.pdf 
8. Artz L. Fear or failure? Why victims of domestic violence retract from the criminal justice process. South African Crime Q. 2011;37. https://doi.org/10.17159/2413-3108/2011/v0i37a855

9. Nuti SV, Wayda B, Ranasinghe I, Wang S, Dreyer RP, Chen SI, et al. The use of google trends in health care research: a systematic review. PLoS One. 2014;9:e109583. https://doi.org/10.1371/journal.pone.0109583 PMid:25337815 PMCid:PMC4215636

10. Tsao SF, Chen $\mathrm{H}$, Tisseverasinghe $\mathrm{T}$, Yang Y, Li L, Butt ZA. What social media told us in the time of COVID-19: a scoping review. Lancet Digit Health. 2021;3:e175-94. https://doi.org/10.1016/S2589-7500(20)30315-0

11. Vosen S, Schmidt T. Forecasting private consumption: surveybased indicators vs. Google trends. Wiley Online Library. 2011;6:565-78. https://doi.org/10.1002/for.1213

12. Fórum Brasileiro de Segurança Pública. Anuário brasileiro de segurança pública 2021 [Internet]. 2020 Oct 19 [cited 2021 Apr 16]. https://forumseguranca.org.br/anuario-brasileiro-segurancapublica/

13. Google Trends. Dia internacional da mulher [Internet]. 2021 Feb 28 [cited 2021 Apr 16].

https://trends.google.com.br/trends/story/BR cu Jmj4q3ABAACuQ M en

14. Viero A, Barbara G, Montisci M, Kustermann K, Cattaneo C. Violence against women in the Covid-19 pandemic: a review of the literature and a call for shared strategies to tackle health and social emergencies. Forensic Sci Int. 2021;319:110650. https://doi.org/10.1016/j.forsciint.2020.110650 PMid:33340849 PMCid:PMC8021946

15. Barros A. Com pandemia, 20 estados têm taxa média de desemprego recorde em 2020 [Internet]. 2021 Mar 10 [cited 2021 
Apr 16]. https://agenciadenoticias.ibge.gov.br/agencianoticias/2012-agencia-de-noticias/noticias/30235-com-pandemia20-estados-tem-taxa-media-de-desemprego-recorde-em-2020

- 16. Power K. The COVID-19 pandemic has increased the care burden of women and families. Sustain Sci Pract Policy. 2020:16:6773. https://doi.org/10.1080/15487733.2020.1776561

- 17. Dang HA, Viet Nguyen C. Gender inequality during the COVID19 pandemic: income, expenditure, savings, and job loss [Internet]. [cited 2021 Jul 12]. https://ftp.iza.org/dp13824.pdf https://doi.org/10.1016/j.worlddev.2020.105296

- 18. Pereira MU, Gaspar RS. Socioeconomic factors associated with reports of domestic violence in large Brazilian cities. Front Public Health. 2021;9:623185.

https://doi.org/10.3389/fpubh.2021.623185 PMid:33604324 PMCid:PMC7884961

\section{Declarações}

Este trabalho foi apoiado pela Organização Panamericana da Saúde (OPAS; SCON2020-00202) e pelo Conselho Nacional de Desenvolvimento Científico e Tecnológico (CNPq; 401542/2020-3) em força tarefa com a Associação Brasileira de Psiquiatria (ABP), Associação Brasileira de Impulsividade e Patologia Dupla (ABIPD) e SAMBE Research Group. 


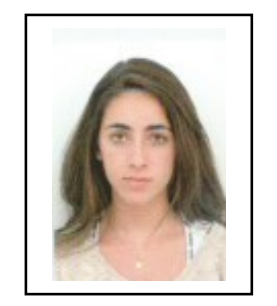

Rafaela Ferreira Guatimosim

$\underline{\text { ORCID } \quad \text { Lattes }}$

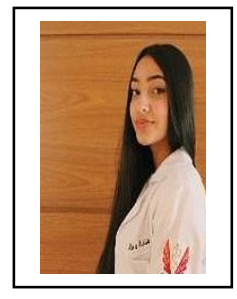

Ana Luiza Silva Teles

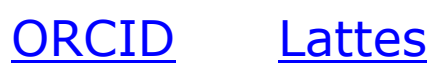

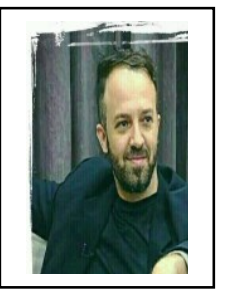

Fabiano Franca Loureiro

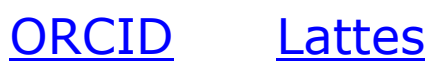

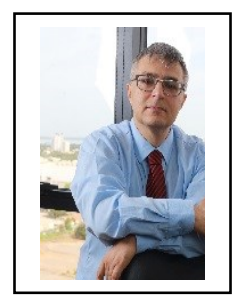

Leonardo Rodrigo Baldaçara

ORCID Lattes

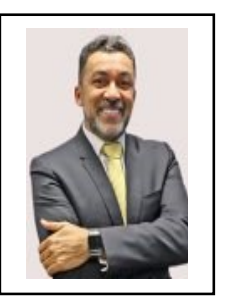

Antônio Geraldo da Silva

$\underline{\text { ORCID Lattes }}$

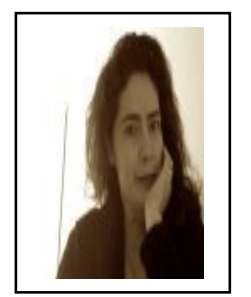

Débora Marques de Miranda $\underline{\text { ORCID Lattes }}$ 
O que sabemos sobre a violência contra as mulheres em tempos pandêmicos?

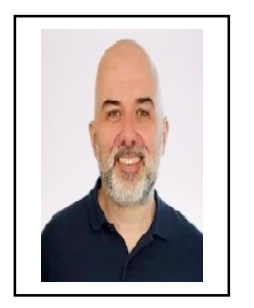

Leandro Fernandes Malloy-Diniz

$\underline{\text { ORCID Lattes }}$ 